\title{
Aquaporin 1 contributes to chondrocyte apoptosis in a rat model of osteoarthritis
}

\author{
HANGFEI GAO ${ }^{1}$, JIANCAO GUI ${ }^{2}$, LIMING WANG $^{2}$, YAN XU $^{2}$, YIQIU JIANG ${ }^{2}$, \\ MINGYUE XIONG ${ }^{1}$ and YONGGUANG CUI ${ }^{1}$
}

\author{
${ }^{1}$ Department of Traumatology, The First Affiliated Hospital and College of Clinical Medicine of \\ Henan University of Science and Technology, Luoyang, Henan 471000; ${ }^{2}$ Department of Orthopaedics, \\ The First Affiliated Hospital of Nanjing Medical University, Nanjing, Jiangsu 210006, P.R. China
}

Received August 5, 2015; Accepted September 19, 2016

DOI: $10.3892 / \mathrm{ijmm} .2016 .2785$

\begin{abstract}
Aquaporins (AQPs) have been found to be associated with a number of diseases. However, the role of AQP-1 in the pathogenesis of osteoarthritis remains unclear. We previously found that AQP-1 expression was upregulated in osteoarthritic cartilage and strongly correlated with caspase- 3 expression and activity. The aim of this study was to further investigate the association of AQP-1 expression with chondrocyte apoptosis in a rat model of osteoarthritis, using RNA interference to knock down AQP-1. For this purspose, 72 male Sprague-Dawley rats were randomly assigned to 3 groups as follows: the control group not treated surgically $(n=24)$, the sham-operated group $(n=24)$, and the osteoarthritis group $(n=24)$. Osteoarthritis was induced by amputating the anterior cruciate ligament and medial collateral ligament and partially excising the medial meniscus. Chondrocytes from the rats with osteoarthritis were isolated and cultured. shRNAs were used to knock down AQP-1 expression in the cultured chondrocytes. The expression of AQP-1 and caspase-3 was determined by reverse transcriptionquantitative polymerase chain reaction. Caspase-3 activity was measured using a caspase- 3 colorimetric assay. The rats in our model of osteoarthritis exhibited severe cartilage damage. The knockdown of AQP-1 decreased caspase-3 expression and activity in the cultured chondrocytes. In addition, the expression of AQP-1 positively correlated with caspase-3 expression and activity. Thus, the findings of our study, suggest that AQP-1 promotes caspase- 3 activation and thereby contributes to chondrocyte apoptosis and to the development of osteoarthritis.
\end{abstract}

\section{Introduction}

Osteoarthritis is a degenerative disease with an irreversible course and serious consequences that affects millions of

Correspondence to: Professor Jiancao Gui, Department of Orthopaedics, The First Affiliated Hospital of Nanjing Medical University, 140 Hanzhong Road, Nanjing, Jiangsu 210006, P.R. China E-mail: guii997@126.com

Key words: osteoarthritis, aquaporin 1, chondrocyte, apoptosis individuals worldwide (1). Increasing age is one of the most common risk factors for osteoarthritis (2). With an increase in life expectancy, more elderly patients are likely to develop osteoarthritis (3), and osteoarthritis is estimated to become the fourth most disabling disease by the year 2020 (1). However, the pathogenesis of the disease remains poorly understood.

Although chondrocytes, which are mainly responsible for the anabolic-catabolic balance in cartilage, account for only $1-2 \%$ of the total cartilage volume, they play an important role in regulating the function of articular cartilage by synthesizing the structural components of the extracellular matrix (ECM) and matrix-degrading proteases (3). Chondrocytes have been found to play a pivotal role in the pathology of osteoarthritis through chondrocyte apoptosis and cartilage matrix degradation (4-6). However, the molecular mechanisms underlying the development of osteoarthritis due to chondrocyte apoptosis have not yet been clearly elucidated.

Aquaporins (AQPs) are specific transmembrane proteins responsible for water transport and are expressed in articular chondrocytes (7). It has been reported that the expression levels of AQPs are associated with apoptosis in many types of cells. However, the role of AQPs in the pathogenesis of osteoarthritis remains unclear $(7,8)$. It remains to be determined whether AQP-1 expression is altered in chondrocytes in osteoarthritis and whether the expression levels of AQP-1 are associated with chondrocyte apoptosis.

We have previously reported that AQP-1 mRNA expression is increased in a rat model of osteoarthritis and positively correlates with the mRNA expression and activity of the the apoptotic marker, caspase-3 (9). In the present study, we further performed RNA interference (RNAi) experiments to knock down AQP-1 and investigated the association between the expression of AQP-1 and the expression and activity of caspase-3. The aim of this study was to further determine the role of AQP-1 expression in chondrocyte apoptosis and to further explore the role of AQP-1 in the pathogenesis of osteoarthritis.

\section{Materials and methods}

Animals. All experimental protocols were approved by the Institutional Animal Care and Use Committee of Nanjing Medical University, Nanjing, China. All procedures were carried 
out in accordance with the Institutional Animal Care and Use Committee Guide at Merck Research Laboratories (Germany). A total of 72 male Sprague-Dawley (SD) rats (8 weeks old, weighing 286-320 g) were obtained from the Animal Center of Nanjing Medical University (Nanjing, China). The animals were housed at room temperature $\left(25^{\circ} \mathrm{C}\right)$ with $60 \%$ humidity and a 12-h light/dark cycle. The animals were fed standard rat chow and were provided with water ad libitum. The rats were randomly assigned to 3 groups as follows: the control group not treated surgically $(n=24)$, the sham-operated group $(n=24)$, and the osteoarthritis group $(\mathrm{n}=24)$.

Establishment of rat model of osteoarthritis. The rats were anesthetized by an intraperitoneal injection of $10 \%$ chloral hydrate $(1-2 \mathrm{ml} / \mathrm{kg})$. The anterior cruciate ligament and medial collateral ligament were cut, and the anterior horn of the medial meniscus was partially removed, via the medial parapatellar approach, as previously described (10). The anterior drawer test and the lateral stress test were used to confirm the dissection of the anterior cruciate ligament and medial collateral ligament. The articular cavity was flushed with iodine and saline. The wounds were sutured, and penicillin $(80,000 \mathrm{U}$; Shanghai Nuotai Chemical Co., Ltd., China) was administered for 3 days. For the rats in the the sham-operated group, the articular cavity was exposed, but the ligaments and anterior horn of the medical meniscus were not removed. For the rats in the the control group, no treatment/surgery was administered. The rats in the each group were forced to move for $2 \mathrm{~h}$ each day by the squirrel wheel method. The general condition of the articular cartilage, based on the color, cracking, softening and osteophyte formation, was observed. The rats were sacrificed by $\mathrm{CO}_{2}$ inhalation, and the knee joints were harvested at 1, 2, 4 and 8 weeks after the osteoarthritis model was established. The samples were stored in $4 \%$ formaldehyde solution at $-80^{\circ} \mathrm{C}$ until use.

Isolation and culture of chondrocytes from rats with osteoarthritis. The cartilage was removed from the rats in the osteoarthritis group at 8 weeks post-surgery and used for chondrocyte isolation and culture. Chondrocytes were isolated from the cartilage matrix by serial digestion with trypsin (Amresco, Solon, OH, USA) and collagenase II (Sigma, St. Louis, MO, USA) and cultured as previously described (11). The survival conditions of the cultured cells were examined under an optical microscope (A11.1535; Opto-Edu, Beijing, China).

Cultured chondrocytes in the logarithmic growth phase were seeded in a 6 -well plate, supplemented with $2 \mathrm{ml}$ Dulbecco's modified Eagle's medium/F-12 medium containing $15 \%$ fetal bovine serum with 200,000 units penicillin. The cells were cultured for 3, 5 days and 1 week in an incubator at $37^{\circ} \mathrm{C}$ with $5 \% \mathrm{CO}_{2}$. The medium was changed every other day. Transfection was performed when the cells reached $80 \%$ confluence.

Hematoxylin and eosin (H\&E) and Alcian blue staining. $\mathrm{H} \& \mathrm{E}$ and Alcian blue staining was used to assess chondrocyte morphology. For H\&E staining (AR1180-100; Boster, Wuhan, China), the cells were stained with H\&E for $5 \mathrm{~min}$. For Alcian blue staining, the cells were stained with Alcian blue, using the Alcian Blue pH 2.5 Stain kit (American MasterTech, Lodi, CA, USA).
Immunofluorescence staining. For immunofluorescence staining, the cells were grown on glass coverslips, rinsed with phosphate-buffered saline (PBS), and fixed in $4 \%$ paraformaldehyde for $30 \mathrm{~min}$ at room temperature. The cells were then permeabilized with $1 \%$ Triton X-100 for $10 \mathrm{~min}$. Following 3 washes with PBS, the cells were incubated with primary antibody against type II collagen (rabbit anti-rat type II collagen, 1:100 dilution; Cat no. 70R-CR008; Fitzgerald Industries International, Acton, MA, USA) at $4^{\circ} \mathrm{C}$ overnight. PBS without primary antibody was used as a negative control. After the primary antibody was removed by washing in PBS, immunoreactivity was detected by incubation with fluorescein-isothiocyanate-conjugated secondary antibody (goat anti-rabbit $\operatorname{IgG}, 1: 100$ dilution; 111-005-144; Jackson Immuno Research, West Grove, PA, USA) at room temperature for $45 \mathrm{~min}$. After the coverslips were washed with PBS, the cells were counterstained with DAPI (Sigma) and examined and photographed under a fluorescence microscope (Olympus Corp., Tokyo, Japan).

RNAi and cell transfection. The rat cDNA sequence (GenBank NM-012778; https://www.ncbi.nlm.nih.gov/ nuccore/NM_012778) was analyzed for potential small interfering RNA (siRNA) target sequences for AQP-1. The oligonucleotide was designed to have a hairpin loop and cloned into the pGenesil-1 plasmid containing the U6 promoter and green fluorescent protein (GFP) (Wuhan Cell Marker Biotechnology Co., Ltd., Wuhan, China). As previously described (12), the AQP-1-shRNA pGenesil-1 plasmid named AQP-1-pGenesil was used for RNAi to knock down AQP-1. The following oligonucleotide was used for AQPI-2 (19 nt): 5'-TTCTCAAA CCACTGGATT-3'. The oligonucleotide used for scrambled shRNA was 5'-GACTTCATAAGGCGCATGC-3'. Chondrocytes at $80 \%$ confluence were transfected with AQP-1-pGenesil using the transfection reagent, Lipofectamine ${ }^{\circledR} 2000$ (Invitrogen Life Technologies, Carlsbad, CA, USA) according to the manufacturer's instructions. Untransfected cells were used as empty controls, and cells transfected with Lipofectamine 2000 were used as the empty Lipofectamie 2000 group. At $48 \mathrm{~h}$ post-transfection, the transfected cells showing GFP expression were sorted using flow cytometry (Guava ${ }^{\circledR}$ easyCyte 8 , Merck Millipore, Billerica, MA, USA) and used in the subsequent experiments.

Reverse transcription-quantitative polymerase chain reaction $(R T-q P C R)$. Total RNA was isolated from the articular cartilage using the TRIzol RNA extraction kit (Invitrogen Life Technologies). RNA was reverse transcribed into complementary DNA (cDNA) using the reverse transcription system (Toyobo Co., Ltd., Osaka, Japan). Quantitative (qPCR) was performed with a $20-\mu 1$ mixture containing $2.5 \mu \mathrm{l} \mathrm{cDNA}$, $0.4 \mu \mathrm{l}$ of each primer, and $10 \mu \mathrm{l} \mathrm{SYBR-Green} \mathrm{(Toyobo} \mathrm{Co.,Ltd.).}$ The following primers were used: 5'-CATTGGCTTGTCTG TGGC-3' (forward) and 5'-TTTGAGAAGTTGCGGGTG-3' (reverse) for AQP-1, 5'-CTGGACTGCGGTATTGAG-3' (forward) and 5'-GGGTGCGGTAGAGTAAGC-3' (reverse) for caspase-3, and 5'-CAAGTTCAACGGCACGTCAA-3' (forward) and 5'-TGGTGAAGACGCCAGAGACTC-3' (reverse) for glyceraldehyde-3-phosphate dehydrogenase (GAPDH). GAPDH was used as an internal control. The 

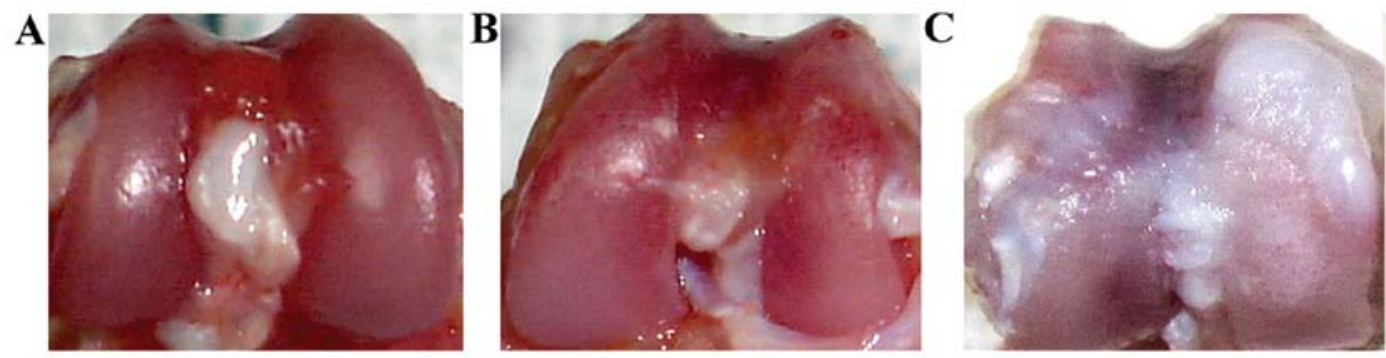

Figure 1. Cartilage damage in rats in (A) the control, (B) sham-operated, and (C) osteoarthritis groups at 8 weeks post-surgery. In the control and sham-operated groups, the surface of the cartilage was resilient and smooth, with no cracks, softening, or osteophyte formation. However, the cartilage of the rats in the osteoarthritis group had lost the original luster and showed obvious roughness, osteophyte formation and cracking. The articular surface also appeared opaque.

reaction conditions were as follows: $95^{\circ} \mathrm{C}$ for $20 \mathrm{sec} ; 50.4^{\circ} \mathrm{C}$ for $20 \mathrm{~min} ; 95^{\circ} \mathrm{C}$ for $60 \mathrm{sec} ; 95^{\circ} \mathrm{C}$ for $15 \mathrm{sec}$ with 40 cycles of $55^{\circ} \mathrm{C}$ for $15 \mathrm{sec}$ and $74^{\circ} \mathrm{C}$ for $45 \mathrm{sec}$. The relative expression levels of AQP-1 and caspase- 3 were calculated using the $2^{-\Delta \Delta C t}$ method, as previously described (13).

Determination of caspase-3 activity. Total protein was extracted from the articular cartilage of the rats in each group or the cultured chondrocytes. Protein concentrations were determined using the BCA Protein Assay kit (Nanjing KeyGen Biotech Co., Ltd., Nanjing, China). Caspase-3 activity was measured using the Caspase-3 Colorimetric Assay kit (Nanjing KeyGen Biotech Co., Ltd.). The plates were read at $405 \mathrm{~nm}$ using a microplate spectrophotometer (Model 680; Bio-Rad, Hercules, CA, USA).

Statistical analysis. Statistical analyses were performed using SPSS 17.0 software (SPSS, Inc., Chicago, IL, USA). Quantitative data are presented as the means \pm standard deviation. One-way analysis of variance (ANOVA) was used to compare the differences among groups, followed by the post hoc Student-Newman-Keuls tests. Pearson's correlation analysis was used to evaluate the association between the expression of AQP-1 and caspase-3 expression or activity. A value of $\mathrm{P}<0.05$ was considered to indicate a statistically significant difference.

\section{Results}

Cartilage damage in our rat model of osteoarthritis. In the control and sham-operated groups, the surface of the cartilage was resilient and smooth, showing no cracks, softening, or osteophyte formation at 8 weeks group (Fig. 1A and B). At 8 weeks post-surgery, synovial hyperplasia was observed in the osteoarthritis group. The cartilage of the rats in the osteoarthritis group had lost the original luster and showed obvious roughness, osteophyte formation and cracking. The articular surface also appeared opaque (Fig. 1C).

Morphology of cultured chondrocytes from rats in our model of osteoarthritis. The chondrocytes isolated from the rats in our model of osteoarthritis were spherical in shape with strong refractivity and became triangular or polygonal in shape following adherence to the culture surfaces. The cell nuclei were round or oval with 1-3 nucleoli and located in the center of the cells. Following culture for 3 days, the cells were clustered and
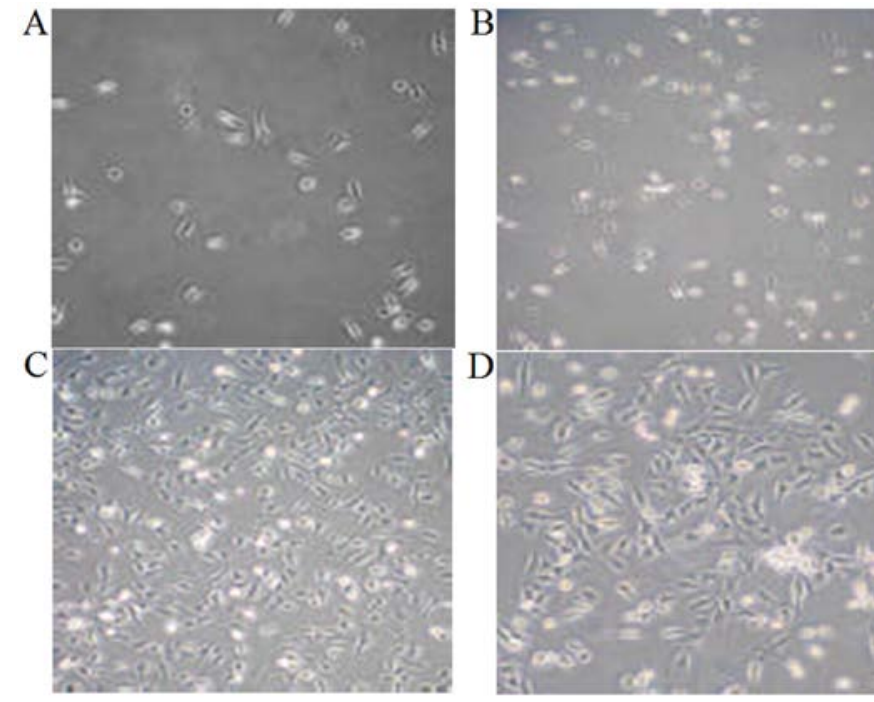

Figure 2. Morphology of cartilage cells at (A) after 1 day of culture, (B) after 3 days of culture, (C) after 5 days of culture, and (D) after 7 days of culture, as shown under an optical microscope (magnification, x100).

grew in a round or oval shape. Following culture for 5 days, the cells overlapped and proliferated significantly to form connections between cells. Matrix materials were deposited around the cells. The cells grew in a monolayer and covered the bottom of the culture bottle following culture for 1 week (Fig. 2).

H\&E staining indicated that the cartilage cells at the 4th passage were triangular or polygonal. The nuclei had double or multiple nucleoli. The ECM was stained red (Fig. 3A and B). Upon Alcian blue staining, the cytoplasm and cell membrane were stained dark blue, suggesting that the cultured chondrocytes synthesized and secreted proteoglycans (Fig. 3C and D). In addition, oositive immunofluorescence staining for type II collagen indicated that chondrocyte-specific type II collagen was mainly distributed in the cytoplasm and cell membrane (Fig. 4A-C).

Knockdown of AQP-1 decreases the expression of caspase-3 in cultured chondrocytes. At $48 \mathrm{~h}$ post-transfection, green fluorescence was clearly observed in the transfected cells (Fig. 4D), indicating the success of the transfection. Flow cytometry revealed that fluorescent cells represented $41.9 \%$ of all cells (Fig. 5B). The expression of AQP-1 was significantly decreased in the cells transfected with AQPI-1-pGenesil-1 


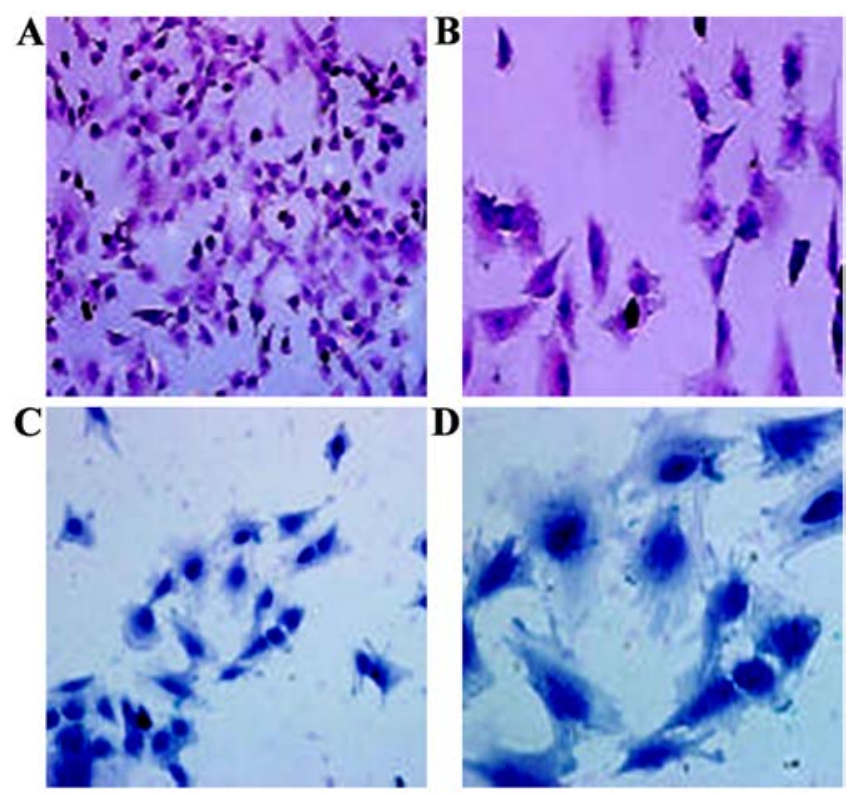

Figure 3. (A and B) Representative images of hematoxylin and eosin staining of cultured chondrocytes from rats with osteoarthritis. (A) Magnification, x200; (B) magnification, $\mathrm{x} 400$. (C and D) Representative images of Alcian blue staining of cultured chondrocytes from rats with osteoarthritis. (C) Magnification, x200; (D) magnification, x400.
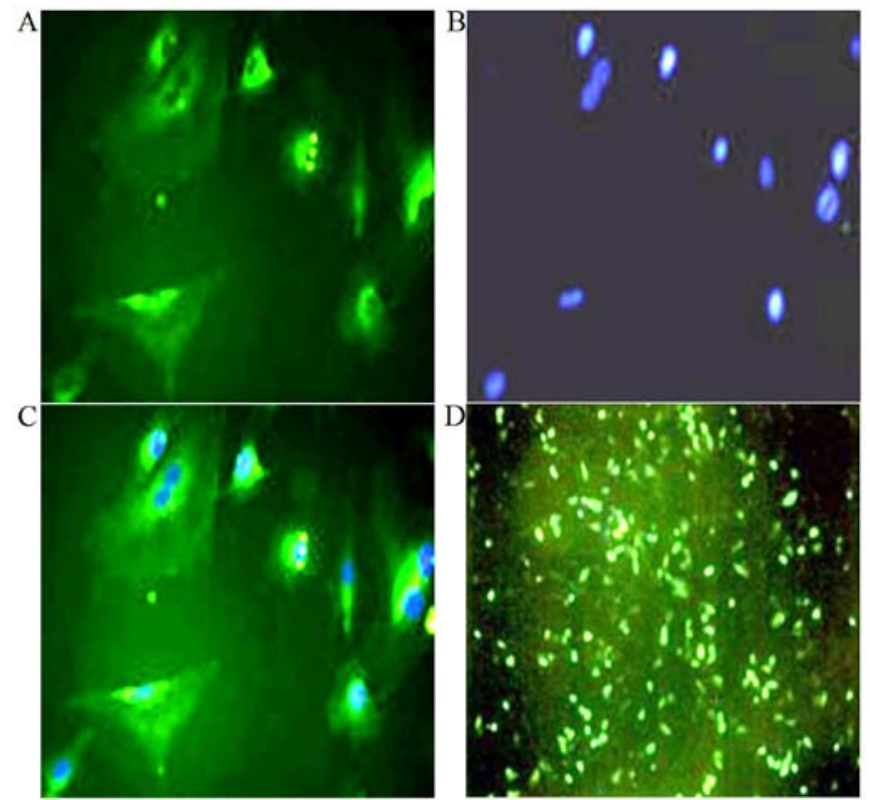

Figure 4. (A-C) Representative images of immunofluorescence staining of type II collagen in cartilage cells (magnification, x200). (A) Type II collagen; (B) DAPI; and (C) merged image. (D) Green fluorescent protein detected by fluorescence microscopy in cultured chondrocytes transfected with AQP-1-pGenesil.
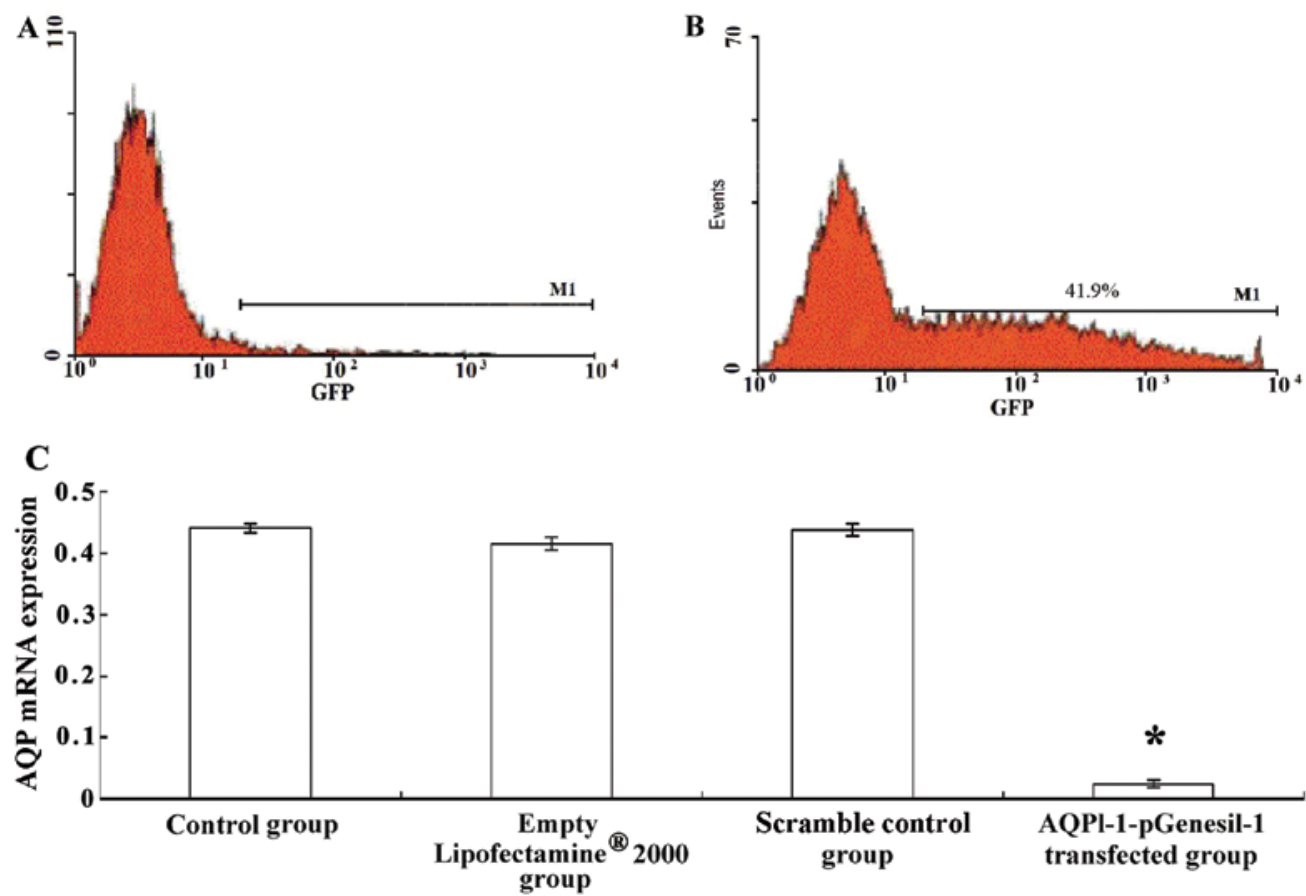

Figure 5. Positive transfection rate detected by flow cytometry. (A) Transfected cartilage cells with (A) no GFP expression; (B) transfected cartilage cells with GFP expression. (C) AQP-1 mRNA expression in control cells, cells treated with Lipofectamine ${ }^{\circledR} 2000$ alone, cells transfected with scrambled shRNA, and cells transfected with AQPl-1-pGenesil-1. " $\mathrm{P}<0.01$ vs normal control group, Lipofectamine ${ }^{\circledR} 2000$ group, and scrambled shRNA group. GFP, green fluorescent protein; $\mathrm{AQP}$, aquaporin.

compared with that in untransfected control cells, cells treated with Lipofectamine ${ }^{\circledR} 2000$ alone, and cells transfected with scrambled shRNA ( $\mathrm{P}<0.01$, Fig. 5C). In addition, the expression and activity of caspase-3 were significantly decreased in the cells transfected with AQPl-1-pGenesil-1 ( $\mathrm{P}<0.05$, Fig. 6A and B).
Correlation between the expression of AQP-1 and caspase-3 expression and activity. Pearson's correlation analysis was used to evaluate the association between the expression of AQP-1 and caspase- 3 expression or activity. The expression of AQP-1 positively correlated with the expression of caspase-3 (Fig. 6C, 
A

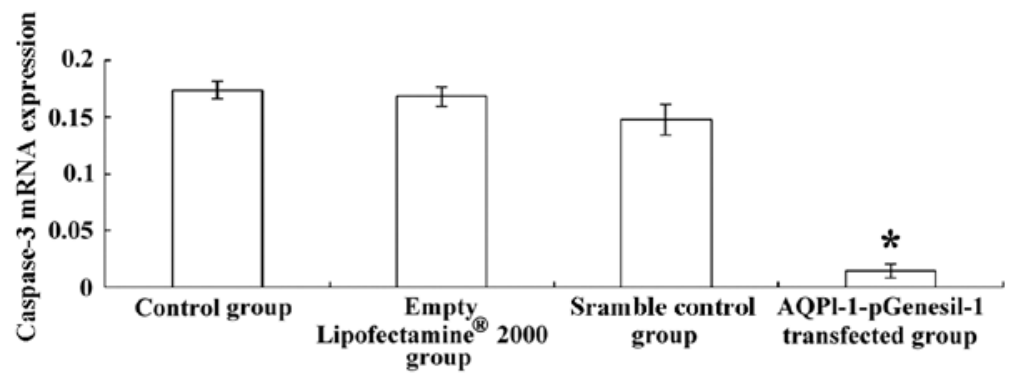

B

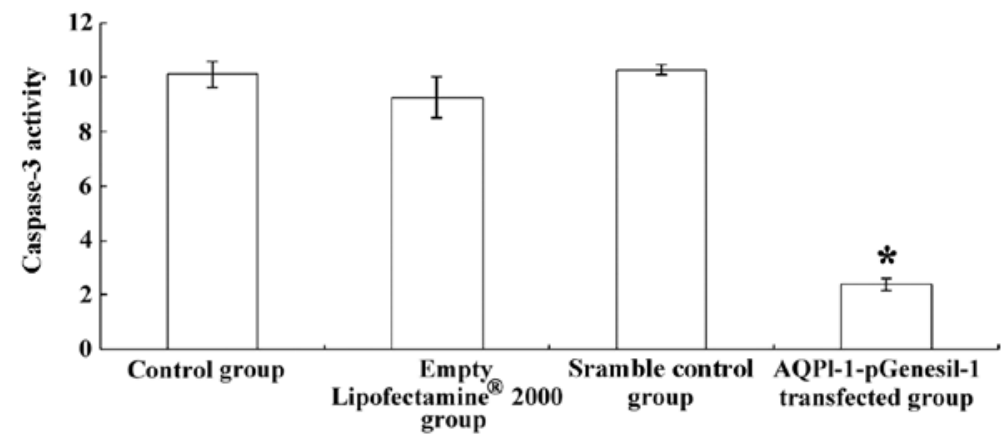

C
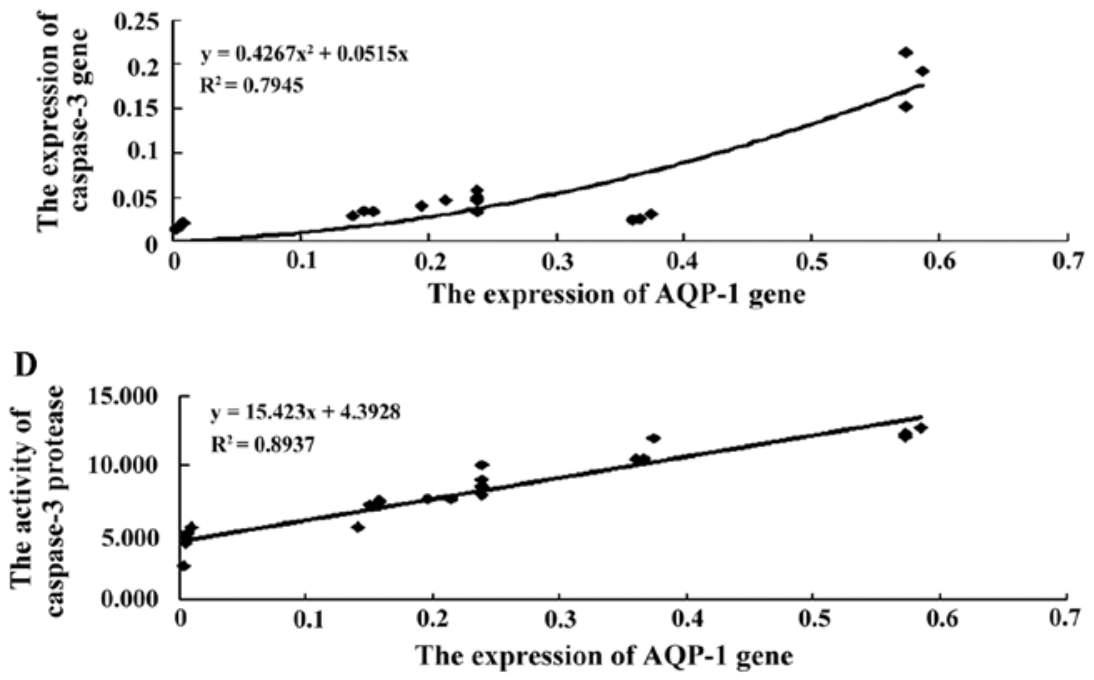

Figure 6. (A) Caspase-3 mRNA expression in control cells, cells treated with Lipofectamine ${ }^{\circledast} 2000$ alone, cells transfected with scrambled shRNA, and cells transfected with AQP1-1-pGenesil-1. "P<0.01 vs. normal control group, Lipofectamine ${ }^{\circledR} 2000$ group, and scrambled shRNA group. (B) Caspase-3 activity in control cells, cells treated with Lipofectamine ${ }^{\circledR} 2000$ alone, cells transfected with scrambled shRNA, and cells transfected with AQPl-1-pGenesil-1. "P<0.01 vs. normal control group, Lipofectamine ${ }^{\circledR} 2000$ group, and scrambled shRNA group. (C) Correlation between the expression of AQP-1 and the expression of caspase-3 and, (D) correlation between the expression of AQP-1 and caspase-3 activity in cultured chondrocytes. (C) Curve fitting was carried out regression analysis using the following equation: $y=0.4267 x^{2}+0.0515 x\left(R^{2}=0.7945, P<0.001\right)$. (D) Curve fitting was carried out regression analysis using the following equation: $\mathrm{y}=15.423 \mathrm{x}+4.3928\left(\mathrm{R}^{2}=0.8937, \mathrm{P}<0.001\right)$.

$\mathrm{r}=0.817 ; \mathrm{P}<0.001)$ and with caspase-3 activity (Fig. $6 \mathrm{C}, \mathrm{r}=0.945$; $\mathrm{P}<0.001)$.

\section{Discussion}

Osteoarthritis is regarded as a pathological condition resulting from the degeneration or destruction of the articular cartilage that covers and protects the moving joints. However, the role of chondrocytes in osteoarthritis remains unclear. We have previously demonstrated that the expression of AQP-1 is upregulated in a rat model of osteoarthritis, accompanied by an increase in caspase-3 expression and activity (9). In the present study, we found that the knockdown of AQP-1 in chondrocytes from rats with osteoarthritis decreased caspase- 3 expression and activity, and that the expression of AQP-1 positively correlated with caspase-3 expression and activity, suggesting that AQP-1 contributes to chondrocyte apoptosis and to the development of osteoarthritis.

Chondrocytes are the major regulators of the process of matrix anabolism and catabolism and, thus, are essential to maintain the homeostasis of the cartilage matrix. Chondrocytes not only synthesize the ECM, but also play a direct role in the degradation process termed as 'chondrocytic chondrolysis'. Injured chondrocytes fail to degrade the damaged matrix in osteoarthritic cartilage and, thus, contribute to the irreversible pathological process of osteoarthritis (3). Chondrocyte apoptosis 
has been found to be a major contributor to the progression of osteoarthritis (14-17). Caspase-3 protease is considered to be a killer protease and a key apoptosis mediator that mediates the terminal phase of apoptosis induced by death receptors or through the mitochondrial pathway (18). Several lines of evidence have indicated that activated caspase- 3 protease will lead to irreversible apoptosis, and thus, the activation of caspase- 3 is regarded as a molecular marker of apoptosis (19-22). In the present study, the significantly upregulated expression of caspase-3 was found in chondrocytes from rats with osteoarthritis, suggesting that chondrocyte apoptosis plays an important role in osteoarthritis.

It has been reported that the apoptotic volume decrease (AVD) is a common apoptotic pathway in various cells of many species and is a common reaction of cells to apoptosis inducers (23). AVD is mainly caused by the flow of monovalent cations though cation channels and alterations in water permeability via AQPs (24-28). It has been demonstrated that water and ion channels play a certain role in cell apoptosis in the central nervous system, and the expression levels of AQPs, potassium channels and chloride channels contribute to the initiation and progression of apoptosis (27). The outflow of water molecules mediated by AQPs has been reported to be one of the preconditions of AVD (25). In addition, the altered expression of AQPs in the mitochondria has been found during the apoptotic process (28). Furthermore, the activation of AQPs followed by mitochondrial swelling has been reported to induce the release of cytochrome $c$ and the activation of caspase enzymes (28). It has also been reported that the overexpression of AQP-1 activates intracellular caspase-3 and induces apoptosis in vitro $(24,25)$. In addition, a decrease in the expression of AQP-1 in hepatocellular carcinoma cells has been shown to be associated with resistance to apoptosis (29). Li et al reported that $\alpha$-melanocyte-stimulating hormone reduced renal tubular epithelial cell apoptosis and prevented the downregulation of AQPs and $\mathrm{Na}^{+}-\mathrm{K}^{+}$ATP enzymes in rats with bilateral ureteral obstruction (30). The inhibition of AQP-1 by mercuric chloride $\left(\mathrm{HgCl}_{2}\right)$ has been reported to induce a decrease in AVD and caspase-3 activity (27). Consistent with the literature, in this study, we found that AQP-1 was upregulated in rats with osteoarthritis, and that the expression of AQP-1 positively correlated with caspase-3 expression and activation in chondrocytes, suggesting that AQP-1 promotes chondrocyte apoptosis via the activation of caspase-3.

The findings that the knockdown of AQP-1 significantly decreased the expression of caspase-3 and activity further confirmed that the upregulation of AQP-1 expression activated caspase-3, and thus contributed to chondrocyte apoptosis and to the development of osteoarthritis.

In conclusion, we previously found that the expression of AQP-1 was upregulated in the osteoarthritic cartilage and that it strongly correlated with caspase-3 expression and activity (9). In the present study, we further found that the inhibition of AQP-1 expression using shRNA decreased the expression of caspase-3 in chondrocytes from rats with osteoarthritis, suggesting that AQP-1 participates in the process of chondrocyte apoptosis, and thereby contributes to the development of osteoarthritis.

\section{Acknowledgements}

The authors appreciate the excellent technical assistance of Dr Haibo Sun (Nanjing Medical University, Nanjing, China).
This study was supported by the special foundation for major scientists of Nanjing City (project no. 3030930).

\section{References}

1. Tesche F and Miosge N: New aspects of the pathogenesis of osteoarthritis: The role of fibroblast-like chondrocytes in late stages of the disease. Histol Histopathol 20: 329-337, 2005.

2. Burnett BP, Levy R and Cole BJ: Metabolic mechanisms in the pathogenesis of osteoarthritis. A review. J Knee Surg 19: 191-197, 2006.

3. Aigner T, Söder S, Gebhard PM, McAlinden A and Haag J: Mechanisms of disease: Role of chondrocytes in the pathogenesis of osteoarthritis - structure, chaos and senescence. Nat Clin Pract Rheumatol 3: 391-399, 2007.

4. Thomas CM, Fuller CJ, Whittles CE and Sharif M: Chondrocyte death by apoptosis is associated with cartilage matrix degradation. Osteoarthritis Cartilage 15: 27-34, 2007.

5. Lee HG and Yang JH: PKC- $\delta$ mediates TCDD-induced apoptosis of chondrocyte in ROS-dependent manner. Chemosphere 81: 1039-1044, 2010.

6. Abouheif MM, Nakasa T, Shibuya H, Niimoto T, Kongcharoensombat W and Ochi M: Silencing microRNA-34a inhibits chondrocyte apoptosis in a rat osteoarthritis model in vitro. Rheumatology (Oxford) 49: 2054-2060, 2010.

7. Trujillo E, González T, Marín R, Martín-Vasallo P, Marples D and Mobasheri A: Human articular chondrocytes, synoviocytes and synovial microvessels express aquaporin water channels; upregulation of AQP1 in rheumatoid arthritis. Histol Histopathol 19: 435-444, 2004.

8. Geyer M, Grässel S, Straub RH, Schett G, Dinser R, Grifka J, Gay S, Neumann E and Müller-Ladner U: Differential transcriptome analysis of intraarticular lesional vs intact cartilage reveals new candidate genes in osteoarthritis pathophysiology. Osteoarthritis Cartilage 17: 328-335, 2009.

9. Gao H, Ren G, Xu Y, Jin C, Jiang Y, Lin L, Wang L, Shen H and Gui L: Correlation between expression of aquaporins 1 and chondrocyte apoptosis in articular chondrocyte of osteoarthritis. Zhongguo Xiu Fu Chong Jian Wai Ke Za Zhi 25: 279-284, 2011 (In Chinese).

10. Hayami T, Pickarski M, Zhuo Y, Wesolowski GA, Rodan GA and Duong LT: Characterization of articular cartilage and subchondral bone changes in the rat anterior cruciate ligament transection and meniscectomized models of osteoarthritis. Bone 38: 234-243, 2006.

11. Sandell LJ, Xing X, Franz C, Davies S, Chang LW and Patra D: Exuberant expression of chemokine genes by adult human articular chondrocytes in response to IL-1beta. Osteoarthritis Cartilage 16: 1560-1571, 2008.

12. Zhang W, Xie CM, Li ZP, Zhu ZW, Yan YS and Du HC: Inhibition of the aquaporin-1 gene expression by RNA interference: experiment with cultured rat pleural mesothelial cells. Zhonghua Yi Xue Za Zhi 87: 1773-1777, 2007 (In Chinese).

13. Livak KJ and Schmittgen TD: Analysis of relative gene expression data using real-time quantitative PCR and the 2(-Delta Delta C(T)) Method. Methods 25: 402-408, 2001.

14. Watrin-Pinzano A, Etienne S, Grossin L, Gaborit N, Cournil-Henrionnet C, Mainard D, Netter P, Gillet P and Galois L: Increased apoptosis in rat osteoarthritic cartilage corresponds to degenerative chondral lesions and concomitant expression of caspase-3. Biorheology 43: 403-412, 2006.

15. Attur MG, Dave M, Akamatsu M, Katoh M and Amin AR: Osteoarthritis or osteoarthrosis: The definition of inflammation becomes a semantic issue in the genomic era of molecular medicine. Osteoarthritis Cartilage 10: 1-4, 2002.

16. Goggs R, Carter SD, Schulze-Tanzil G, Shakibaei M and Mobasheri A: Apoptosis and the loss of chondrocyte survival signals contribute to articular cartilage degradation in osteoarthritis. Vet J 166: 140-158, 2003.

17. Kim HA, Lee YJ, Seong SC, Choe KW and Song YW: Apoptotic chondrocyte death in human osteoarthritis. J Rheumatol 27: 455-462, 2000.

18. Nicholson DW and Thornberry NA: Caspases: Killer proteases. Trends Biochem Sci 22: 299-306, 1997.

19. Kliem H, Berisha B, Meyer HH and Schams D: Regulatory changes of apoptotic factors in the bovine corpus luteum after induced luteolysis. Mol Reprod Dev 76: 220-230, 2009. 
20. Irony-Tur-Sinai $M$, Lichtenstein $M$, Brenner $T$ and Lorberboum-Galski H: IL2-caspase3 chimeric protein controls lymphocyte reactivity by targeted apoptosis, leading to amelioration of experimental autoimmune encephalomyelitis. Int Immunopharmacol 9: 1236-1243, 2009.

21. Schwarz K, Simonis G, Yu X, Wiedemann S and Strasser RH: Apoptosis at a distance: Remote activation of caspase-3 occurs early after myocardial infarction. Mol Cell Biochem 281: 45-54, 2006.

22. Earnshaw WC, Martins LM and Kaufmann SH: Mammalian caspases: Structure, activation, substrates, and functions during apoptosis. Annu Rev Biochem 68: 383-424, 1999.

23. Maeno E, Ishizaki Y, Kanaseki T, Hazama A and Okada Y: Normotonic cell shrinkage because of disordered volume regulation is an early prerequisite to apoptosis. Proc Natl Acad Sci USA 97: 9487-9492, 2000.

24. Jablonski EM and Hughes FM Jr: The potential role of caveolin-1 in inhibition of aquaporins during the AVD. Biol Cell 98: 33-42, 2006.

25. Flamenco P, Galizia L, Rivarola V, Fernandez J, Ford P and Capurro C: Role of AQP2 during apoptosis in cortical collecting duct cells. Biol Cell 101: 237-250, 2009.

26. Jablonski EM, Webb AN, McConnell NA, Riley MC and Hughes FM Jr: Plasma membrane aquaporin activity can affect the rate of apoptosis but is inhibited after apoptotic volume decrease. Am J Physiol Cell Physiol 286: C975-C985, 2004.
27. Jessica Chen M, Sepramaniam S, Armugam A, Shyan Choy M, Manikandan J, Melendez AJ, Jeyaseelan K and Sang Cheung N: Water and ion channels: Crucial in the initiation and progression of apoptosis in central nervous system? Curr Neuropharmacol 6: 102-116, 2008.

28. Lee WK, Bork U, Gholamrezaei F and Thévenod F: $\mathrm{Cd}(2+)$-induced cytochrome c release in apoptotic proximal tubule cells: Role of mitochondrial permeability transition pore and $\mathrm{Ca}(2+)$ uniporter. Am J Physiol Renal Physiol 288: F27-F39, 2005.

29. Jablonski EM, Mattocks MA, Sokolov E, Koniaris LG, Hughes FM Jr, Fausto N, Pierce RH and McKillop IH: Decreased aquaporin expression leads to increased resistance to apoptosis in hepatocellular carcinoma. Cancer Lett 250: 36-46, 2007.

30. Li C, Shi Y, Wang W, Sardeli C, Kwon TH, Thomsen K, Jonassen T, Djurhuus JC, Knepper MA, Nielsen S and Frøkiaer J: alpha-MSH prevents impairment in renal function and dysregulation of AQPs and Na-K-ATPase in rats with bilateral ureteral obstruction. Am J Physiol Renal Physiol 290: F384-F396, 2006. 